\title{
Sublobar resection is associated with improved outcomes over radiotherapy in the management of high-risk elderly patients with Stage I non-small cell lung cancer: a systematic review and meta-analysis
}

\author{
Huan-Huan Wang ${ }^{1, *}$, Chun-Ze Zhang ${ }^{2,}{ }^{*}$, Bai-Lin Zhang ${ }^{1}$, Jie Chen ${ }^{1}$, Xian-Liang Zeng ${ }^{1}$, \\ Lei Deng ${ }^{3}$, Mao-Bin Meng ${ }^{1}$ \\ ${ }^{1}$ Department of Radiation Oncology, Tianjin's Clinical Research Center for Cancer and Key Laboratory of Cancer Prevention \\ and Therapy, Tianjin Medical University Cancer Institute and Hospital, National Clinical Research Center for Cancer, Tianjin \\ 300060, China \\ ${ }^{2}$ Department of Colorectal Surgery, Tianjin Union Medical Center, Tianjin 300121, China \\ ${ }^{3}$ Department of Thoracic Cancer and Huaxi Student Society of Oncology Research, West China Hospital, West China School \\ of Medicine, Sichuan University, Sichuan Province 610041, China \\ *These authors contributed equally to this work
}

Correspondence to: Mao-Bin Meng, email: mmeng@tmu.edu.cn

Keywords: non-small cell lung cancer, radiotherapy, sublobar resection, overall survival, pattern of failure

Received: October 04, $2016 \quad$ Accepted: December 12, $2016 \quad$ Published: December 17, 2016

\section{ABSTRACT}

Background and Aim: A matched-pair comparison was performed to compare the efficacy and safety of sublobar resection versus radiotherapy for high-risk elderly patients with Stage I non-small cell lung cancer (NSCLC).

Patients and Methods: We searched the Cochrane Library, MEDLINE, CENTRAL, EMBASE and manual searches. The meta-analysis was performed to compare overall survival, pattern of failure, and toxicity among the homogeneous studies. Subdivided analyses were also performed.

Results: Sixteen studies containing 11540 patients were included in the meta-analysis. Among these studies, 9 were propensity-score matched (PSM) cohort studies, and 7 were cohort studies. Sublobar resection, compared with radiotherapy (either conventional fraction radiation therapy or stereotactic body radiation therapy), significantly improved the overall survival regardless in both PSM and non-PSM analyses (all $p<0.05$ ). However, the difference in the pattern of failure and toxicity were not significant (all $p>0.05$ ).

Conclusions: Sublobar resection was associated with improved outcomes in high-risk elderly patients with Stage I NSCLC, which supports the need to compare both treatments in large prospective, randomized, controlled clinical trials.

\section{INTRODUCTION}

Anatomic resection (lobectomy or pneumonectomy) with systematic lymph node dissection or sampling is the mainstay of therapy in early-stage non-small cell lung cancer (NSCLC) [1-2]. However, more than half of patients are not candidates for the standard surgical procedure, most often because they are elderly or have pulmonary dysfunction, poor performance status, or comorbidities [3]. In these compromised patients, various treatment strategies are available including observation, conventional fractionated radiation therapy (CFRT), stereotactic body radiation therapy (SBRT), radiofrequency ablation, or sublobar resection (SLR, i.e. wedge resection or segmentectomy) with or without systematic lymph node dissection [2, 4-5].

The majority of compromised patients with early-stage NSCLC who are not eligible for lobectomy should be offered an alternative local treatment, as more than half of patients with inoperable cancer who do not undergo treatment will die of disease [6-8]. Radiofrequency ablation is an emerging modality for the treatment of inoperable stage I NSCLC; however, data 
for radiofrequency ablation are less mature because of small sample size, undefined adaptive tumor volumes, and shorter follow-ups [11-13]. CFRT used for the local treatment of early-Stage NSCLC patients with poor pulmonary reserve has been used reluctantly due to poor local control and lung toxicity [9-10]. Therefore, the two most optimal modalities for compromised Stage I NSCLC patients appear to be SLR and SBRT according to the European Organization for Research and Treatment of Cancer and the International Society of Geriatric Oncology [14].

Both SLR and SBRT are associated with excellent local control rates, and they also have shown the capacity to maintain pulmonary function post-procedure [14]. However, the question remains as to which intervention is the best treatment for compromised patients who cannot tolerate lobectomy. To resolve this issue, an intergroup randomized trial (RTOG 1021/ACOSOG Z4099) comparing SLR with SBRT in high-risk patients with Stage I NSCLC was initiated [15]. Unfortunately, it was closed in May 2013 due to slow patient enrollment. The aim of this study was to conduct a meta-analysis to evaluate the efficacy and safety of treatments for high-risk elderly patients with Stage I NSCLC, with comparison between two treatment groups: SLR versus radiotherapy (either CFRT or SBRT). It is anticipated that this metaanalysis will provide evidence-based information for clinical practice.

\section{RESULTS}

\section{Search results}

The systematic literature search identified a total of 3792 relevant references. After careful reading of the titles and abstracts, 3769 references were excluded because the objective did not satisfy the inclusion criteria. A total of 23 studies were retrieved for further assessment. Of these, five studies were excluded because of an inability to obtain the SLR data [16-20] and two studies were excluded because of duplication [21-22]. Ultimately, 16 studies (11,540 patients) [23-38] met our inclusion criteria.

\section{Common characteristics}

A majority of the included studies were conducted in the USA, except for two studies [23, 38]. All the patients were diagnosed with Stage I NSCLC, and the median age was 66 years and older. A PSM comparison was performed in 9 studies, and a total of 10,870 matched patients were included [25, 27-28, 31-35, 37]. Five studies evaluated the efficacy and safety of SLR versus CFRT for these patients [23-27]; 10 studies were designed to compare SLR versus SBRT [28-37]; and one study evaluated the efficacy and safety of SLR versus CFRT and SBRT [38], as shown in Table 1.
For patients undergoing SLR, the main operation was thoracotomy or video-assisted thoracoscopic surgery (VATS) with or without systematic lymph node dissection or sampling. The dosage range in the CFRT group was 45.0-90.3 Gy in 22-40 fractions, and that in the SBRT group was 30-66 Gy in 2-8 fractions. The treatment characteristics of the included studies are listed in Table 2.

\section{Survival outcome and patterns of failure}

The included studies presented Kaplan-Meier curves, numbers of events, and 1-, 2-, 3-, and 5-year OS rates. In these studies, 5782 patients were treated with SLR, 429 patients were treated with CFRT, and 5329 patients were treated with SBRT. We compared the ORs for OS and patterns of failure between SLR and radiotherapy either CFRT or SBRT.

SLR, compared with radiotherapy either CFRT or SBRT, led to significantly better the 1-, 2-, 3-, and 5-year OS rates regardless of whether the analysis was PSM or non-PSM (all $p<0.05$ ). When we recalculated the results after excluding one study because had considerable weight [47], we found that SLR, compared with SBRT, was associated with significantly better 3- and 5-year OS rates in the PSM analyses (all $p<0.05$ ). However, the difference in the pattern of failure and toxicity were not significant (all $p>0.05$ ) (Tables 3 and 4).

\section{Heterogeneity analysis and publication bias}

There was evidence of heterogeneity for OS and pattern of failure (Tables 3 and 4). L'Abbé plots of 3-year OS of SLR versus SBRT showed evidence of heterogeneity (Figure 1A). However, a review of funnel plots could not rule out the potential for publication bias for either analysis. Publication bias was not evident when the Begg rank correlation method and Egger's Weighted regression method ( $p=0.75$ for 3 -year OS and $p=0.46$ for local failure) were used for SLR versus SBRT (Figure 1B).

\section{Sensitivity analysis}

A sensitivity analysis was performed to explore the influence of study quality on the effect size. In the primary analysis, outcomes of 3-year OS and local failure for SLR versus SBRT were applied in a random-effects model. In terms of 3-year OS, when we recalculated the sensitivity analysis after application of a fixed-effects model, we found that the overall estimates were virtually identical and the CIs were similar between the sensitivity analysis $(\mathrm{OR}=2.80 ; 95 \%$ CI 2.53-3.09; $p=0.001$ ) and the meta-analysis $(\mathrm{OR}=2.91 ; 95 \%$ CI $1.94-4.38$; $p=0.0001)$. In addition, we found that the OR and $95 \%$ CI for local failure were also similar $(\mathrm{OR}=0.87$; $95 \%$ CI $0.50-1.51 ; p=0.62)$ and $(\mathrm{OR}=0.83 ; 95 \%$ CI $0.23-3.02 ; p=0.78)$. 
Table 1: Main characteristics of all the included studies

\begin{tabular}{|c|c|c|c|c|c|c|c|c|c|c|c|c|c|c|c|}
\hline \multicolumn{3}{|c|}{ Study } & \multicolumn{3}{|c|}{ Main characteristics } & \multicolumn{4}{|c|}{ Overall survival } & \multicolumn{6}{|c|}{ Patterns of failure (No. of patients) } \\
\hline Author (Year) & Country & Design & Arms & No. of Patients & Age & 1-year & 2 -year & 3-year & 5 -year & LF & RF & LDF & RDF & DF & LRF \\
\hline SLR or WR versus CFR' & & & & & & & & & & & & & & & \\
\hline Yano T (1995) ${ }^{23}$ & Japan & Cohort & $\begin{array}{l}\text { CFRT } \\
\text { SLR }\end{array}$ & $\begin{array}{l}18 \\
17\end{array}$ & $\begin{array}{l}74.5 \\
68.5\end{array}$ & $\begin{array}{l}72.8 \% \\
95.7 \%\end{array}$ & $\begin{array}{l}63.4 \% \\
95.7 \%\end{array}$ & $\begin{array}{l}14.4 \% \\
61.5 \%\end{array}$ & $\begin{array}{c}14.4 \% \\
55 \%\end{array}$ & $\begin{array}{l}4 \\
3\end{array}$ & NA & NA & NA & NA & NA \\
\hline Ghosh S (2003) ${ }^{24}$ & UK & Cohort & $\begin{array}{l}\text { CHART } \\
\text { WR }\end{array}$ & $\begin{array}{l}19 \\
47\end{array}$ & $\begin{array}{l}76.9 \\
76.6\end{array}$ & $\begin{array}{l}80 \% \\
98 \%\end{array}$ & $\begin{array}{l}80 \% \\
90 \%\end{array}$ & $\begin{array}{l}68.4 \% \\
86.4 \%\end{array}$ & $\begin{array}{l}39 \% \\
74 \%\end{array}$ & $\begin{array}{l}5 \\
9\end{array}$ & NA & $\begin{array}{c}6 \\
13\end{array}$ & NA & $\begin{array}{l}1 \\
4\end{array}$ & NA \\
\hline Yendamuri S (2007) ${ }^{25}$ & USA & $\begin{array}{c}\text { Cohort } \\
\text { (Matched pairs) }\end{array}$ & $\begin{array}{l}\text { 3D-CRT } \\
\text { WR }\end{array}$ & $\begin{array}{l}34 \\
34\end{array}$ & $\begin{array}{l}72 \\
72\end{array}$ & $\begin{array}{l}86.2 \% \\
91.7 \%\end{array}$ & $\begin{array}{c}80 \% \\
82.2 \%\end{array}$ & $\begin{array}{l}58.3 \% \\
65.4 \%\end{array}$ & $\begin{array}{l}38.7 \% \\
41.5 \%\end{array}$ & NA & $\mathrm{NA}$ & NA & NA & NA & $\mathrm{NA}$ \\
\hline Fernandez FG (2012) ${ }^{27}$ & USA & $\begin{array}{c}\text { Cohort } \\
\text { (Matched pairs) }\end{array}$ & $\begin{array}{l}\text { 3D-CRT } \\
\text { SLR }\end{array}$ & $\begin{array}{l}319 \\
319\end{array}$ & $\begin{array}{c}77 \\
75.5\end{array}$ & $\begin{array}{l}77.4 \% \\
87.5 \%\end{array}$ & $\begin{array}{c}52 \% \\
71.6 \%\end{array}$ & $\begin{array}{l}41 \% \\
52 \%\end{array}$ & $\begin{array}{l}20 \% \\
41 \%\end{array}$ & NA & NA & NA & NA & NA & NA \\
\hline SLR or WR versus SBR & & & & & & & & & & & & & & & \\
\hline Forquer JA (2009) ${ }^{28}$ & USA & $\begin{array}{c}\text { Cohort } \\
\text { (Matched pairs) }\end{array}$ & $\begin{array}{l}\text { SBRT } \\
\text { SLR }\end{array}$ & $\begin{array}{l}19 \\
19\end{array}$ & $\begin{array}{l}66 \\
67\end{array}$ & NA & NA & NA & NA & $\begin{array}{l}0 \\
2\end{array}$ & $\begin{array}{l}4 \\
3\end{array}$ & $\begin{array}{l}1 \\
1\end{array}$ & $\begin{array}{l}0 \\
1\end{array}$ & $\begin{array}{l}1 \\
0\end{array}$ & $\begin{array}{l}4 \\
5\end{array}$ \\
\hline Grills IS $(2010)^{29}$ & USA & Cohort & $\begin{array}{l}\text { SBRT } \\
\text { WR }\end{array}$ & $\begin{array}{l}58 \\
69\end{array}$ & $\begin{array}{l}78 \\
74\end{array}$ & $\begin{array}{l}81.7 \% \\
98.2 \%\end{array}$ & $\begin{array}{l}74.5 \% \\
92 \%\end{array}$ & $\begin{array}{c}56 \% \\
81.3 \%\end{array}$ & $\begin{array}{c}56 \% \\
52.4 \%\end{array}$ & $\begin{array}{c}3 \\
14\end{array}$ & $\begin{array}{c}3 \\
13\end{array}$ & $\begin{array}{l}14 \\
29\end{array}$ & $\begin{array}{l}14 \\
28\end{array}$ & $\begin{array}{l}11 \\
15\end{array}$ & $\begin{array}{c}6 \\
27\end{array}$ \\
\hline Varlotto J (2013) $)^{31}$ & USA & $\begin{array}{c}\text { Cohort } \\
\text { (Matched pairs) }\end{array}$ & $\begin{array}{l}\text { SBRT } \\
\text { WR }\end{array}$ & $\begin{array}{c}137 \\
48\end{array}$ & $\begin{array}{l}73.3 \\
67.5\end{array}$ & $\begin{array}{l}68.2 \% \\
100 \%\end{array}$ & $\begin{array}{l}50.8 \% \\
94.1 \%\end{array}$ & $\begin{array}{l}42.3 \% \\
86.3 \%\end{array}$ & $\begin{array}{l}31.7 \% \\
86.3 \%\end{array}$ & NA & $\mathrm{NA}$ & $\mathrm{NA}$ & NA & $\begin{array}{c}21 \\
4\end{array}$ & $\begin{array}{c}15 \\
6\end{array}$ \\
\hline Shirvani SM $(2012)^{32}$ & USA & $\begin{array}{c}\text { Cohort } \\
\text { (Matched pairs) }\end{array}$ & $\begin{array}{l}\text { SBRT } \\
\text { SLR }\end{array}$ & $\begin{array}{l}112 \\
112\end{array}$ & $\begin{array}{l}75 \\
75\end{array}$ & $\begin{array}{l}80.2 \% \\
81.9 \%\end{array}$ & $\begin{array}{l}61.4 \% \\
63.7 \%\end{array}$ & $\begin{array}{l}56.7 \% \\
60.5 \%\end{array}$ & NA & NA & NA & NA & NA & NA & NA \\
\hline Port JL (2014) ${ }^{33}$ & USA & $\begin{array}{c}\text { Cohort } \\
\text { (Matched pairs) }\end{array}$ & $\begin{array}{c}\text { SBRT } \\
\mathrm{WR} \pm \mathrm{BT}\end{array}$ & $\begin{array}{l}23 \\
76\end{array}$ & $\begin{array}{l}76 \\
72\end{array}$ & NA & NA & $\begin{array}{l}75 \% \\
87 \%\end{array}$ & NA & $\begin{array}{l}1 \\
1\end{array}$ & $\begin{array}{l}2 \\
1\end{array}$ & $\begin{array}{l}5 \\
3\end{array}$ & $\begin{array}{l}6 \\
3\end{array}$ & $\begin{array}{l}4 \\
2\end{array}$ & $\begin{array}{l}3 \\
2\end{array}$ \\
\hline Matsuo Y (2014) ${ }^{34}$ & Japan & $\begin{array}{c}\text { Cohort } \\
\text { (Matched pairs) }\end{array}$ & $\begin{array}{l}\text { SBRT } \\
\text { SLR }\end{array}$ & $\begin{array}{l}53 \\
53\end{array}$ & $\begin{array}{l}76 \\
76\end{array}$ & $\begin{array}{l}94.5 \% \\
94.2 \%\end{array}$ & $\begin{array}{c}80 \% \\
83.5 \%\end{array}$ & $\begin{array}{l}61.2 \% \\
72.9 \%\end{array}$ & $\begin{array}{l}40.4 \% \\
55.6 \%\end{array}$ & NA & NA & NA & NA & NA & NA \\
\hline Puri V (2015) ${ }^{35}$ & USA & $\begin{array}{c}\text { Cohort } \\
\text { (Matched pairs) }\end{array}$ & $\begin{array}{l}\text { SBRT } \\
\text { SLR }\end{array}$ & $\begin{array}{l}4555 \\
4555\end{array}$ & $\begin{array}{l}73.8 \\
73.7\end{array}$ & $\begin{array}{l}87.1 \% \\
89 \%\end{array}$ & $\begin{array}{l}62.4 \% \\
76.3 \%\end{array}$ & $\begin{array}{c}47 \% \\
61.7 \%\end{array}$ & $\begin{array}{l}26.9 \% \\
43.1 \%\end{array}$ & NA & NA & NA & NA & NA & NA \\
\hline Parashar B (2015) ${ }^{36}$ & USA & Cohort & $\begin{array}{l}\text { SBRT } \\
\text { WR }\end{array}$ & $\begin{array}{c}97 \\
123\end{array}$ & $\begin{array}{l}77 \\
77\end{array}$ & $\begin{array}{l}96.2 \% \\
100 \%\end{array}$ & $\begin{array}{l}91.5 \% \\
100 \%\end{array}$ & $\begin{array}{l}91.5 \% \\
97.7 \%\end{array}$ & $\begin{array}{l}89.6 \% \\
97.7 \%\end{array}$ & $\begin{array}{c}4 \\
10\end{array}$ & $\mathrm{NA}$ & $\begin{array}{l}22 \\
32\end{array}$ & NA & $\begin{array}{l}18 \\
22\end{array}$ & NA \\
\hline Paul S (2016) ${ }^{37}$ & USA & $\begin{array}{c}\text { Cohort } \\
\text { (Matched pairs) }\end{array}$ & $\begin{array}{l}\text { SBRT } \\
\text { SLR }\end{array}$ & $\begin{array}{l}201 \\
201\end{array}$ & $\begin{array}{l}77.6 \\
75.6\end{array}$ & $\begin{array}{l}90.5 \% \\
92.5 \%\end{array}$ & $\begin{array}{r}69 \% \\
81.3 \%\end{array}$ & $\begin{array}{l}57.4 \% \\
67.2 \%\end{array}$ & $\begin{array}{c}27.8 \% \\
62 \%\end{array}$ & NA & NA & NA & NA & NA & $\mathrm{NA}$ \\
\hline Safi S $(2015)^{38 \uparrow}$ & Germany & Cohort & $\begin{array}{c}\text { CFRT or SBRT } \\
\text { SLR }\end{array}$ & $\begin{array}{l}49 \\
42\end{array}$ & $\begin{array}{l}73.5 \\
69.6\end{array}$ & $\begin{array}{l}94 \% \\
93 \%\end{array}$ & $\begin{array}{l}69 \% \\
85 \%\end{array}$ & $\begin{array}{l}18 \% \\
66 \%\end{array}$ & NA & $\begin{array}{c}17 \\
4\end{array}$ & $\mathrm{NA}$ & NA & NA & NA & NA \\
\hline
\end{tabular}

Among 49 patients receiving RT, 28 and 21 patients have received CFRT and SBRT, respectively.

Abbreviations: CHART: continuous hyperfractionated accelerated radiotherapy; CFRT: conventional fractionated radiotherapy; SLR: sublobar resection; LF: local failure; RF: regional failure; LDF: local and distant failure; RDF: regional and distant failure; DF: distant failure; LRF: locoregional failure; WR: wedge resection; BT: brachytherapy; mths: months; 3D-CRT: three-dimensional conformal radiotherapy; NA: not reported.

\section{DISCUSSION}

To our knowledge, this study represents the only available quantitative assessment of published data on SLR versus radiotherapy either CFRT or SBRT for highrisk elderly patients with Stage I NSCLC. The present

A Begger's funnel plot with pseudo 95\% Cls (3-year OS)

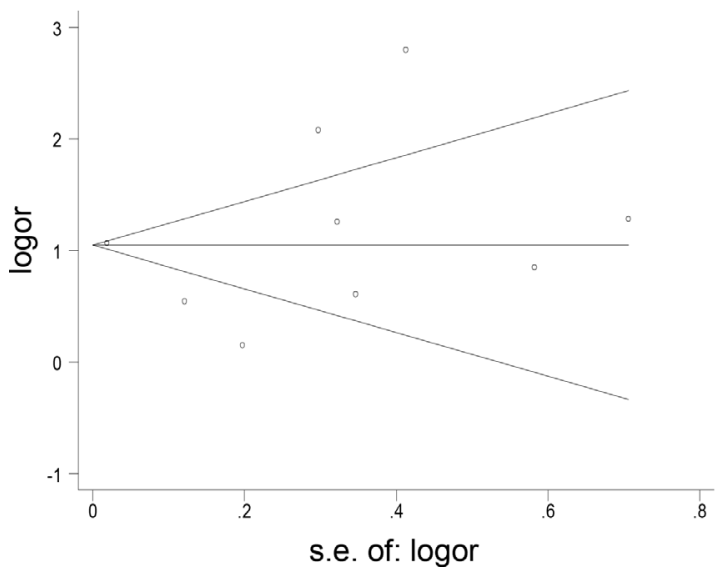

study revealed that SLR was associated with a better OS compared with radiotherapy either CFRT or SBRT. Although such studies have some limitations, together they contain credible evidence that the administration of each treatment modality is worthy of additional study. It is hoped that this will help to better define the roles of

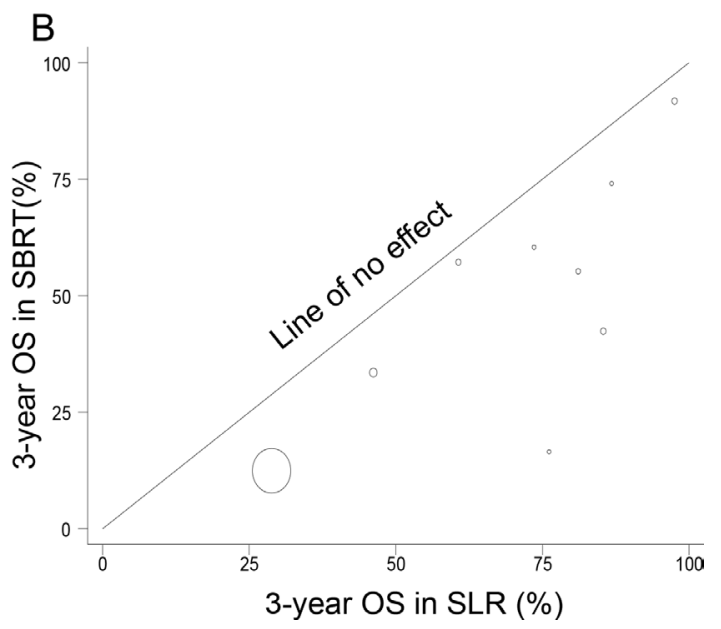

Figure 1: Analyses of publication bias and heterogeneity. (A) The funnel plot appears symmetric, and there was no evidence of publication bias for SLR versus SBRT using the Bagg rank correlation method ( $p=0.75$ for 3 -year OS). The horizontal line in the funnel plot indicates the fixed-effects summary estimate, and the sloping lines indicate the expected $95 \%$ CIs for a given standard error, assuming no heterogeneity between studies. (B) L'Abbé plot showing the 3-year OS rates, comparing the effect size in SBRT and SLR. 
Table 2: Technical features of SLR, CFRT, and SBRT for treatment of high-risk elderly patients with stage I NSCLC

\begin{tabular}{|c|c|c|c|c|c|c|c|}
\hline Study & \multicolumn{2}{|c|}{ SLR or WR } & \multicolumn{5}{|c|}{ CFRT or SBRT } \\
\hline Author (Year) & Resection type & MLN dissection & $\begin{array}{c}\text { Prescription } \\
\text { dose }(G y)\end{array}$ & $\begin{array}{c}\text { No. of } \\
\text { fractions }\end{array}$ & $\begin{array}{c}\text { Does per } \\
\text { fraction }(\mathrm{Gy})\end{array}$ & $\mathrm{BED}_{10}(\mathrm{~Gy})$ & Isodose line $(\%)$ \\
\hline \multicolumn{8}{|l|}{ SLR or WR versus CFRT } \\
\hline Yano T $(1995)^{23}$ & Thoracotomy & NA & $51.2,60,70,80$ & $30-40$ & 1.6 or 2.0 & $59.39,72,84,96$ & $95 \%$ \\
\hline Ghosh S $(2003)^{24}$ & Thoracotomy & Cervical medianstinoscopy & 54 & 36 & 1.5 & 62.1 & $95 \%$ \\
\hline Yendamuri S $(2007)^{25}$ & VATS or Thoracotomy & $\begin{array}{l}\text { MLN dissection or } \\
\text { sampling }\end{array}$ & $66(45-90.3)$ & 22 & 3 & 85.8 & $95 \%$ \\
\hline Hsie M $(2009)^{26}$ & VATS or Thoracotomy & None & $70(60-75)$ & 28 & $2.5(2.0-4.11)$ & 87.5 & $95 \%$ \\
\hline Fernandez FG $(2012)^{27}$ & Thoracotomy & \pm MLN sampling & NA & NA & NA & NA & $95 \%$ \\
\hline \multicolumn{8}{|l|}{ SLR or WR versus SBRT } \\
\hline Forquer JA $(2009)^{28}$ & Thoracotomy & \pm MLN sampling & 60 or 66 & 3 & 20 or 22 & 180 or 211.2 & 80 \\
\hline Grills IS $(2010)^{29}$ & VATS or Thoracotomy & $\begin{array}{l} \pm \text { Mediastinoscopy or } \\
\text { MLN dissection }\end{array}$ & 48 or 60 & 4 or 5 & 12 & 105.6 or 132 & $80(60-90)$ \\
\hline Parashar B $(2010)^{30}$ & Thoracotomy & None & $30-60$ & $2-4$ & $10-15$ & $60-150$ & 100 \\
\hline Varlotto J $(2013)^{31}$ & Thoracotomy & \pm MLN sampling & $48-60$ & $3-5$ & 12,20 & $67.2,78,90$ & NA \\
\hline Shirvani SM $(2012)^{32}$ & Thoracotomy & \pm MLN sampling & NA & NA & NA & NA & NA \\
\hline Port JL $(2014)^{33}$ & VATS or Thoracotomy & \pm MLN sampling & $48(30-60)$ & $4(3-5)$ & $12(6-20)$ & NA & 100 \\
\hline Matsuo Y $(2014)^{34}$ & VATS or Thoracotomy & \pm MLN sampling & $48,56,60$ & $4,4,8$ & $12,14,7.5$ & $105.6,134.4,105$ & NA \\
\hline Puri V $(2015)^{35}$ & VATS or Thoracotomy & \pm MLN sampling & $53.83 \pm 6.78$ & NA & NA & NA & NA \\
\hline Parashar B $(2015)^{36}$ & Thoracotomy & Mediastinoscopy & $48(30-60)$ & $4(3-5)$ & $12(10-12)$ & 105.6 & 100 \\
\hline Paul S $(2016)^{37}$ & VATS or Thoracotomy & \pm MLN sampling & NA & NA & NA & NA & NA \\
\hline Safi S $(2015)^{38 \dagger}$ & VATS or Thoracotomy & $\begin{array}{l}\text { MLN dissection or } \\
\text { sampling }\end{array}$ & $\begin{array}{l}\text { CFRT: } 66 \\
\text { SBRT: } 45 \\
\text { (median) }\end{array}$ & $\begin{array}{l}\text { CFRT: } 33 \\
\text { SBRT: } 3 \\
\text { (median) }\end{array}$ & $\begin{array}{l}\text { CFRT: } 2 \\
\text { SBRT: } 18 \\
\text { (median) }\end{array}$ & $\begin{array}{l}\text { CFRT: } 79.2 \\
\text { SBRT: NA }\end{array}$ & $\begin{array}{l}95 \% \\
\text { NA }\end{array}$ \\
\hline
\end{tabular}

${ }^{\dagger}$ Among 49 patients receiving RT, 28 and 21 patients have received CFRT and SBRT, respectively.

Abbreviations: CFRT: conventional fractionated radiotherapy; SLR: sublobar resection; MLN: mediastinal lymph node; Gy: Gray; BED: biologically equivalent dose; VATS:

video-assisted thoracoscopic surgery; SBRT: stereotactic body radiation oncology; NA: not reported.

these therapies for high-risk elderly patients with Stage I NSCLC.

It was noted that SLR was included segmentectomy and wedge resection in this study. Theoretically, segmentecomy is considered superior ontologically than wedge resection because it provides a larger parenchymal margin and an increased nodal yield [39-40]. For example, Ezer $\mathrm{N}$ et al. assessed the efficacy of SBRT versus segmentecomy or wedge resection separately using SEER database, and found that SBRT treated patients had significantly worse OS and lung cancer-specific OS compared with patients treated with segmentecomy. Nevertheless, OS and lung cancerspecific OS after wedge resection and SBRT were not significantly different [41]. However, some studies have shown that lobectomy and segmentectomy for small clinical Stage I NSCLC are equivalent, whereas wedge resection showed inferior outcomes [42-44]. Consistent with our results and these findings, further studies are warranted for SBRT versus SLR stratified by segmentecomy or wedge resection.

Since SBRT does not intentionally treat lymphatic nodal basins, nodal staging is of critical importance. In this study, the majority of included studies reported that PETCT was used for patients who received SBRT. PET-CT staging of NSCLC has been shown to have a sensitivity of $85 \%$ and specificity of $90 \%$ [45]. Low rates of isolated nodal failure for NSCLC after PET-CT staging of 2.3\%$10 \%$ are reported in the literature [46-47]. In practice, SLR and SBRT can be used to address the primary tumor, but do not treat the mediastinal or intralobar lymph nodes. As PET-CT and other imaging modalities continue to more accurately assess the status of the lymph nodes in NSCLC, the relevance of highly targeted treatments, such as SBRT, for stage I NSCLC patients will further increase.

Treatment-related toxicity is an important factor in selecting and appropriate therapy. Seven studies $[24,27-28,31-32,35,37]$ in this study reported adverse events. SLR, CFRT, and SBRT have specific complications. CFRT and SBRT cause toxicity to the normal structures that surround the tumor, resulting in esophagitis, pneumonitis, hemoptysis, and chest wall pain, but no deaths were attributed to CFRT or SBRT. SLR can affect patients' quality of life in different ways such as further impairment of pulmonary function and chronic pain such as arrhythmia, myocardial infarction, pneumonia, thoracic empyema, and severe lung hemorrhage. In addition, it was noted that whether elderly patients face an increased risk of complications following SBRT for early-Stage NSCLC, as has been reported following surgery. Mancini et al. recently demonstrated that elderly patients undergoing SBRT for early-Stage NSCLC appear to have similar risk of toxicity and rate of efficacy as in younger patients [48].

We believe PSM analysis was performed to reduce selection bias between patients with SLR and SBRT. In this study, PSM accounted for factors of age, gender, tumor stage, pathology, lung function, ECOG performance score, race, comorbidity index i.e. Charlson Comorbidity Index [27, 29, 31-34, 36, 38], and tumor grade, etc. 
Table 3: SLR versus CFRT or SBRT for high-risk elderly stage I NSCLC: a meta-analysis of OS

\begin{tabular}{|c|c|c|c|c|c|c|c|}
\hline End point & $\begin{array}{l}\text { No. of } \\
\text { studies }\end{array}$ & $\begin{array}{c}\text { No. of } \\
\text { patients }\end{array}$ & OR & $95 \%$ CI & Significance & Publication bias & Heterogeneity \\
\hline \multicolumn{8}{|l|}{ SLR versus CFRT } \\
\hline \multicolumn{8}{|l|}{ 1-year survival } \\
\hline All studies combined & 5 & 891 & 2.30 & $1.57-3.37$ & 0.0001 & 0.46 & 0.54 \\
\hline PSM analysis & 2 & 706 & 2.01 & $1.34-3.03$ & 0.001 & & 0.87 \\
\hline None PSM analysis & 3 & 185 & 6.20 & $1.88-20.50$ & 0.003 & & 1.00 \\
\hline \multicolumn{8}{|l|}{ 2-year survival } \\
\hline All studies combined & 5 & 891 & 2.11 & $1.59-2.81$ & 0.0001 & 0.31 & 0.11 \\
\hline PSM analysis & 2 & 706 & 2.10 & $1.54-2.87$ & 0.0001 & & 0.05 \\
\hline None PSM analysis & 3 & 185 & 2.18 & $1.08-4.40$ & 0.03 & & 0.15 \\
\hline \multicolumn{8}{|l|}{ 3-year survival } \\
\hline All studies combined & 5 & 891 & 1.54 & $1.18-2.01$ & 0.002 & 0.81 & 0.28 \\
\hline PSM analysis & 2 & 706 & 1.53 & $1.14-2.06$ & 0.005 & & 0.71 \\
\hline None PSM analysis & 3 & 185 & 1.59 & $0.87-2.91$ & 0.14 & & 0.09 \\
\hline \multicolumn{8}{|l|}{ 5-year survival } \\
\hline All studies combined & 5 & 891 & 2.73 & $2.02-3.69$ & 0.0001 & 0.81 & 0.67 \\
\hline PSM analysis & 2 & 706 & 2.80 & $1.99-3.94$ & 0.0001 & & 0.86 \\
\hline None PSM analysis & 3 & 185 & 2.50 & $1.32-4.72$ & 0.05 & & 0.32 \\
\hline \multicolumn{8}{|l|}{ SLR versus SBRT } \\
\hline \multicolumn{8}{|l|}{ 1-year survival } \\
\hline All studies combined & 8 & 10465 & 1.64 & $1.02-2.64$ & 0.04 & 1.00 & 0.05 \\
\hline PSM analysis & 5 & 10027 & 1.44 & $1.32-1.57$ & 0.0001 & & 0.16 \\
\hline $\begin{array}{l}\text { PSM analysis excluding } \\
\text { reference } 50\end{array}$ & 4 & 917 & 1.63 & $0.65-4.09$ & 0.29 & & 0.04 \\
\hline None PSM analysis & 3 & 438 & 4.62 & $1.66-12.86$ & 0.003 & & 0.06 \\
\hline \multicolumn{8}{|l|}{ 2-year survival } \\
\hline All studies combined & 8 & 10465 & 1.90 & $1.20-3.02$ & 0.006 & 1.00 & 0.0001 \\
\hline PSM analysis & 5 & 10027 & 1.95 & $1.26-3.02$ & 0.003 & & 0.003 \\
\hline $\begin{array}{l}\text { PSM analysis excluding } \\
\text { reference } 50\end{array}$ & 4 & 917 & 2.15 & $0.98-4.75$ & 0.06 & & 0.002 \\
\hline None PSM analysis & 3 & 438 & 9.01 & $3.88-20.93$ & 0.0001 & & 0.08 \\
\hline \multicolumn{8}{|l|}{ 3-year survival } \\
\hline All studies combined & 9 & 10564 & 2.91 & $1.94-4.38$ & 0.0001 & 0.75 & 0.0001 \\
\hline PSM analysis & 6 & 10126 & 2.29 & $1.48-3.55$ & 0.0001 & & 0.0001 \\
\hline $\begin{array}{l}\text { PSM analysis excluding } \\
\text { reference } 50\end{array}$ & 5 & 1016 & 2.17 & $1.21-3.87$ & 0.009 & & 0.007 \\
\hline None PSM analysis & 3 & 438 & 5.63 & $3.22-9.86$ & 0.0001 & & 0.05 \\
\hline \multicolumn{8}{|l|}{ 5-year survival } \\
\hline All studies combined & 6 & 10588 & 2.97 & $1.51-5.83$ & 0.002 & 1.00 & 0.0001 \\
\hline PSM analysis & 4 & 9803 & 3.74 & $1.92-7.26$ & 0.0001 & & 0.003 \\
\hline $\begin{array}{l}\text { PSM analysis excluding } \\
\text { reference } 50\end{array}$ & 3 & 693 & 3.55 & $1.06-11.94$ & 0.04 & & 0.002 \\
\hline None PSM analysis & 2 & 347 & 1.83 & $0.37-9.13$ & 0.46 & & 0.03 \\
\hline
\end{tabular}

Abbreviations: CFRT: conventional fractionated radiotherapy; PSM: propensity-score matched; SBRT: stereotactic body radiation therapy; SLR: sublobar resection; OR: Odds ratio; CI: confidence interval.

Importantly, we found that compared with SBRT, SLR significant improved OS in the PSM analysis; however, the pattern of failure after SLR was similar to that after SBRT (Tables 3 and 4).

Some limitations of the present study must be acknowledged. First, there was no randomization, and the comparison was subject to bias. Second, some studies did not perform PSM meta-analysis may be influence the outcome. Third, the radiographic definitions of failure were probably different between the patients treated with SLR and those treated with radiotherapy. After SLR, there would be no visible tumor left behind, whereas 
Table 4: SLR versus CFRT or SBRT for high-risk elderly Stage I NSCLC: a meta-analysis of pattern of failures

\begin{tabular}{|c|c|c|c|c|c|c|c|}
\hline End point & $\begin{array}{l}\text { No. of } \\
\text { studies }\end{array}$ & $\begin{array}{c}\text { No. of } \\
\text { patients }\end{array}$ & OR & $95 \%$ CI & Significance & $\begin{array}{c}\text { Publication } \\
\text { bias }\end{array}$ & Heterogeneity \\
\hline \multicolumn{8}{|l|}{ SLR versus CFRT } \\
\hline LF & 3 & 185 & 1.09 & $0.50-2.36$ & 0.84 & 1.00 & 0.69 \\
\hline $\mathrm{RL}$ & 1 & 84 & 0.82 & $0.20-3.33$ & 0.79 & & \\
\hline$\overline{\mathrm{DF}}$ & 2 & 150 & 0.74 & $0.27-1.98$ & 0.54 & & 0.84 \\
\hline LRF & 1 & 84 & 0.72 & $0.26-1.98$ & 0.53 & & \\
\hline \multicolumn{8}{|l|}{ SLR versus SBRT } \\
\hline \multicolumn{8}{|l|}{ LF } \\
\hline All studies combined & 6 & 622 & 0.83 & $0.23-3.02$ & 0.78 & 0.46 & 0.008 \\
\hline PSM analysis & 2 & 137 & 0.68 & $0.11-4.31$ & 0.68 & & 0.16 \\
\hline None PSM analysis & 4 & 485 & 0.83 & $0.17-3.96$ & 0.81 & 0.73 & 0.003 \\
\hline \multicolumn{8}{|l|}{$\mathrm{RF}$} \\
\hline All studies combined & 3 & 264 & 1.08 & $0,66-7.03$ & 0.94 & 0.30 & 0.03 \\
\hline PSM analysis & 2 & 137 & 2.29 & $0.60-8.81$ & 0.23 & & 0.28 \\
\hline None PSM analysis & 1 & 127 & 0.23 & $0.06-0.87$ & 0.03 & & \\
\hline \multicolumn{8}{|l|}{$\mathrm{DF}$} \\
\hline All studies combined & 6 & 716 & 1.36 & $0.88-2.11$ & 0.16 & 0.09 & 0.25 \\
\hline PSM analysis & 3 & 322 & 2.79 & $1.10-7.06$ & 0.03 & & 0.44 \\
\hline None PSM analysis & 3 & 394 & 1.05 & $0.63-1.76$ & 0.84 & & 0.55 \\
\hline \multicolumn{8}{|l|}{ LRF } \\
\hline All studies combined & 4 & 449 & 0.77 & $0.22-2.47$ & 0.69 & 0.73 & 0.007 \\
\hline PSM analysis & 3 & 322 & 1.12 & $0.53-2.40$ & 0.76 & & 0.18 \\
\hline None PSM analysis & 1 & 127 & 0.18 & $0.07-0.48$ & 0.001 & & \\
\hline
\end{tabular}

Abbreviations: CFRT: conventional fractionated radiotherapy; PSM: propensity-score matched; SBRT: stereotactic body radiation therapy; SLR: sublobar resection; OR: Odds ratio; CI: confidence interval; LF: local failure; RF: regional failure; DF: distant failure; LRF: locoregional failure.

after radiotherapy, a visible tumor frequently persists and local control is defined as no growth. In addition, some differences between SLR and radiotherapy were observed. Histological confirmation of NSCLC was performed before treatment for all cases receiving radiotherapy, but not SLR. However, patients underwent surgery have pathological staging, but not radiotherapy.

The choice of treatment for high-risk elderly patients with early-Stage NCSLC should be made based on several variables such as patient characteristics, tumor characteristics, and local practice. The development of a personalized treatment model to determine the best treatment for high-risk elderly patients with early-Stage NCSLC based on several such characteristics might be the next step in the treatment of these patients. It will be beneficial to define the impact of each treatment modality on patient care in terms of cost, survival, and improvement in quality of life and to determine the optimal combination therapy for effective palliation and cure of high-risk elderly patients with early-Stage NSCLC.

In conclusion, the findings of this meta-analysis suggest that SLR was associated with better overall survival compared over radiotherapy either CFRT or SBRT in the management of high-risk elderly patients with
Stage I NSCLC. Considering the strength of the evidence, additional randomized controlled trials are needed before each treatment modality can be recommended routinely.

\section{MATERIALS AND METHODS}

\section{Criteria for inclusion}

Acceptable publications met the following criteria: 1) patients with Stage I NSCLC; 2 ) age $>65$ years; 3 ) a treatment group receiving SLR with or without systematic lymph node dissection or sampling and a control group receiving radiotherapy either CFRT or SBRT; and 4) reported data for overall survival (OS) or patterns of failure for calculation of the odds ratios (ORs) and 95\% confidence intervals (CIs).

\section{Exclusion criteria}

Studies were excluded if they did not meet the criteria above and 1) involved animal studies or in vitro studies; 2) did not represent primary research (reviews, editorials, commentaries, case reports, and letters to the editor); 3) represented duplicate publications of other 
studies previously identified in our systematic evaluation; or 4) investigated toxicity only, without OS or pattern of failures, were also excluded.

\section{Search strategy}

Retrieval of trials was performed through the Cochrane Library, MEDLINE, CENTRAL, and EMBASE. The search was designed to initially find all trials involving the terms: "non-small cell lung cancer" or "non-small cell lung carcinoma" or "carcinoma, non-small cell lung" and "sublobar resection" or "SLR" or "wedge resection" or "segmentectomy" or "segmental resection" and "radiation therapy" or "radiotherapy" or "RT" or "stereotactic body radiation therapy" or "stereotactic radiotherapy" or "stereotactic body radiotherapy" or "stereotactic body radiosurgery" or "stereotactic ablative body radiotherapy" or "stereotactic ablative radiotherapy" or "SBRT" or "SABR" (and multiple synonyms for each term). We also manually searched the general reviews on NSCLC and references from published clinical trials. The search results were downloaded to a reference database and screened further.

\section{Outcome measurements}

Outcome measurements of these trials comprised: 1) OS; 2) patterns of failure; and 3) adverse events. Survival included 1-, 2-, 3-, and 5-year survival rates, which were extracted from actual numbers reported in the trials or derived from the survival curves. The lacked key information for calculation with methods developed by Parma et al. [49], Williamson et al. [50], and Tierney et al. [51].

Patterns of failure included local failure, regional failure, distant metastasis, and locoregional failure. Local failure included primary tumor recurrence and recurrence in the involved lobe; regional failure was defined as tumor recurrence in the ipsilateral uninvolved lobe or ipsilateral hilar and mediastinal nodes; and distant metastasis was defined as any disease in contralateral nodes or distant sites. Primary tumor recurrence was diagnosed on the basis of histologic confirmation or enlargement of the local tumor on CT that continued for at least 6 months. Positron emission tomography (PET)-CT was considered when primary tumor recurrence was highly suspected [52-53].

\section{Review methods}

\section{Data extraction}

Three reviewers independently selected the trials and performed the data extraction. Discrepancies were resolved by discussion among reviewers. Information lacking in the original publications was supplemented through correspondence with the original principal investigator. Finally, the following information was extracted from each included trial: 1) the characteristics of the study; 2) the number of patients allocated and patient characteristics; 3 ) the interventional measures used (CFRT, SBRT, and SLR); and 4) outcomes such as OS, patterns of failure, and adverse events.

\section{Statistical methods}

We performed the meta-analysis by pooling CFRT or SBRT and SLR data for an overall analysis regardless of study design. To determine the influence of the PSM analysis on the conclusions of the meta-analysis, subgroup analysis was conducted [54]. Pooled ORs were presented as standard plots with 95\% CIs. All $p$-values were two-sided, and $p<0.05$ was considered statistically significant.

In addition, inter-trial heterogeneity in treatment effect was evaluated using both the Q statistic test and a visual display in a L'Abbé plot [55]. Second, a randomeffects model was employed using the DerSimonian and Laird (DL) method [56] to calculate 95\% CIs, resulting in wider intervals and, thus, a more conservative estimate of treatment effects compared with a fixed-effects model using the Mentel-Haenszel (MH) method. For trials in which the constructed $2 \times 2$ tables contained cells with zero events, a standard correction factor of 0.5 was added to each cell. Third, we applied two different statistical models (a fixed-effects model and a random-effects model) to perform the sensitivity analysis in accordance with the recommendations from the Cochrane Collaboration and the Quality of Reporting of Meta-analysis guidelines. Finally, Begg and Mazumdar's proposed adjusted rank correlation test [57] and Egger's linear regression approach [58] were used to measure publication bias $(p<0.05$ was considered representative of statistically significant publication bias), which was shown as a funnel plot. Analysis was performed using the statistical software Intercooled Stata version 8.2 for Windows (Stata Corporation, College Station, TX, USA).

\section{ACKNOWLEDGMENTS}

No benefits in any form have been or will be received from a commercial party directly or indirectly related to the subject of this article. We thank all principal investigators of the included studies from the literature review in the current study. In particular, we would like to acknowledge the work of Drs. Yano T, Ghosh S, Yendamuri S, Hsie M, Fernandez FG, Forquer JA, Grills IS, Parashar B, Varlotto J, Shirvani SM, Port JL, Matsuo Y, Puri V, Parashar B, Paul S, and Safi $S$. Furthermore, we thank the anonymous referee for his/her very helpful comments, which remarkably improved the quality of this paper.

\section{CONFLICTS OF INTEREST}

We have no conflicts of interests. 


\section{FUNDING}

This work was supported by the Tianjin Health and Family Planning Commission Foundation (No. 2014KR14).

\section{Authors' contributions}

All authors have read and approved the manuscript. We have no financial disclosures. We are not using any copyrighted information in this paper. No text, text boxes, figures, or tables in this article have been previously published or owned by another party.

\section{REFERENCES}

1. Howington JA, Blum MG, Chang AC, Balekian A, Murthy SC. Treatment of stage I and II non-small cell lung cancer: Diagnosis and management of lung cancer, 3rd ed: American College of Chest Physicians evidence-based clinical practice guidelines. Chest. 2013; 143:e278S-313S.

2. NCCN Clinical Practice Guidelines in Non-small cell lung cancer (Version 1. 2016). Available at www.nccn.org.

3. Potosky AL, Saxman S, Wallace RB, Lynch CF. Population variations in the initial treatment of non-small-cell lung cancer. J Clin Oncol. 2004; 22:3261-3268.

4. Fernando HC, Schuchert M, Landreneau R, Daly BT. Approaching the high-risk patient: sublobar resection, stereotactic body radiation therapy, or radiofrequency ablation. Ann Thorac Surg. 2010; 89:S2123-S2127.

5. Johnson DH, Schiller JH, Bunn PA Jr. Recent clinical advances in lung cancer management. J Clin Oncol. 2014; 32:973-982.

6. McGarry RC, Song G, des Rosiers P, Timmerman R. Observation-only management of early stage, medically inoperable lung cancer: poor outcome. Chest. 2002; 121:1155-1158.

7. Raz DJ, Zell JA, Ou SH, Gandara DR, Anton-Culver H, Jablons DM. Natural history of stage I non-small cell lung cancer: implications for early detection. Chest. 2007; 132:193-199.

8. Powell JW, Dexter E, Scalzetti EM, Bogart JA. Treatment advances for medically inoperable non-small-cell lung cancer: emphasis on prospective trials. Lancet Oncol. 2009; 10:885-894.

9. Abratt RP, Morgan GW. Lung toxicity following chest irradiation in patients with lung cancer. Lung Cancer. 2002; 35:103-109.

10. Fang LC, Komaki R, Allen P, Guerrero T, Mohan R, Cox JD. Comparison of outcomes for patients with medically inoperable Stage I non-small-cell lung cancer treated with two-dimensional vs. three-dimensional radiotherapy. Int J Radiat Oncol Biol Phys. 2006; 66:108-116.

11. Pennathur A, Luketich JD, Abbas G, Chen M, Fernando HC, Gooding WE, Schuchert MJ, Gilbert S, Christie NA,
Landreneau RJ. Radiofrequency ablation for the treatment of stage I non-small cell lung cancer in high-risk patients. J Thorac Cardiovasc Surg. 2007; 134:857-864.

12. Nguyen CL, Scott WJ, Young NA, Rader T, Giles LR, Goldberg M. Radiofrequency ablation for the treatment of stage I non-small cell lung cancer in high-risk patients. Chest. 2005; 128:3507-3511.

13. Lencioni R, Crocetti L, Cioni R, Suh R, Glenn D, Regge D, Helmberger T, Gillams AR, Frilling A, Ambrogi M, Bartolozzi C, Mussi A. Response to radiofrequency ablation of pulmonary tumours: a prospective, intention-to-treat, multicentre clinical trial (the RAPTURE study). Lancet Oncol. 2008; 9:621-628.

14. Pallis AG, Gridelli C, Wedding U, Faivre-Finn C, Veronesi G, Jaklitsch M, Luciani A, O'Brien M. Management of elderly patients with NSCLC; updated expert's opinion paper: EORTC Elderly Task Force, Lung Cancer Group and International Society for Geriatric Oncology. Ann Oncol. 2014; 25:1270-1283.

15. Fernando HC, Timmerman R. American College of Surgeons Oncology Group Z4099/Radiation Therapy Oncology Group 1021: a randomized study of sublobar resection compared with stereotactic body radiotherapy for high-risk stage I non-small cell lung cancer. J Thorac Cardiovasc Surg. 2012; 144:S35-S38.

16. Louie AV, Rodrigues G, Hannouf M, Zaric GS, Palma DA, Cao JQ, Yaremko BP, Malthaner R, Mocanu JD. Stereotactic body radiotherapy versus surgery for medically operable Stage I non-small-cell lung cancer: a Markov model-based decision analysis. Int J Radiat Oncol Biol Phys. 2011; 81:964-973.

17. Palma D, Visser O, Lagerwaard FJ, Belderbos J, Slotman B, Senan S. Treatment of stage I NSCLC in elderly patients: a population-based matched-pair comparison of stereotactic radiotherapy versus surgery. Radiother Oncol. 2011; 101:240-244.

18. Puri V, Crabtree TD, Kymes S, Gregory M, Bell J, Bradley JD, Robinson C, Patterson GA, Kreisel D, Krupnick AS, Meyers BF. A comparison of surgical intervention and stereotactic body radiation therapy for stage I lung cancer in high-risk patients: a decision analysis. J Thorac Cardiovasc Surg. 2012; 143:428-436.

19. Crabtree TD, Puri V, Robinson C, Bradley J, Broderick S, Patterson GA, Liu J, Musick JF, Bell JM, Yang M, Meyers BF. Analysis of first recurrence and survival in patients with stage I non-small cell lung cancer treated with surgical resection or stereotactic radiation therapy. J Thorac Cardiovasc Surg. 2014; 147:1183-1191.

20. Kastelijn EA, El Sharouni SY, Hofman FN, Van Putte BP, Monninkhof EM, Van Vulpen M, Schramel FM. Clinical Outcomes in Early-stage NSCLC Treated with Stereotactic Body Radiotherapy Versus Surgical Resection. Anticancer Res. 2015; 35:5607-5614.

21. Shirvani SM, Jiang J, Chang JY, Welsh J, Likhacheva A, Buchholz TA, Swisher SG, Smith BD. Lobectomy, sublobar 
resection, and stereotactic ablative radiotherapy for earlystage non-small cell lung cancers in the elderly. JAMA Surg. 2014; 149:1244-1253.

22. Forquer JA, McGarry RC, Kesler KA, Juliar BE, Watson C, Harkenrider M, Kruter L. A matched-pair analysis of sublobar resection and stereotactic body radiotherapy for stage I NSCLC. Int J Radiat Oncol Biol Phys. 2007; 69:S492-S493.

23. Yano T, Yokoyama H, Yoshino I, Tayama K, Asoh H, Hata K, Ichinose Y. Results of a limited resection for compromised or poor-risk patients with clinical stage I nonsmall cell carcinoma of the lung. J Am Coll Surg. 1995; 181:33-37.

24. Ghosh S, Sujendran V, Alexiou C, Beggs L, Beggs D. Long term results of surgery versus continuous hyperfractionated accelerated radiotherapy (CHART) in patients aged $>70$ years with stage 1 non-small cell lung cancer. Eur J Cardiothorac Surg. 2003; 24:1002-1007.

25. Yendamuri S, Komaki RR, Correa AM, Allen P, Wynn B, Blackmon S, Hofstetter WL, Rice DC, Roth JA, Swisher SG, Vaporciyan AA, Walsh GL, Mehran RJ. Comparison of limited surgery and three-dimensional conformal radiation in high-risk patients with stage I nonsmall cell lung cancer. J Thorac Oncol. 2007; 2:1022-1028.

26. Hsie M, Morbidini-Gaffney S, Kohman LJ, Dexter E, Scalzetti EM, Bogart JA. Definitive treatment of poorrisk patients with stage I lung cancer: a single institution experience. J Thorac Oncol. 2009; 4:69-73.

27. Fernandez FG, Crabtree TD, Liu J, Meyers BF. Sublobar resection versus definitive radiation in patients with stage IA non-small cell lung cancer. Ann Thorac Surg. 2012; 94:354-360.

28. Forquer JA, Fakiris AJ, McGarry RC, Cheung MK, Watson C, Harkenrider M, Henderson MA, Lo SS. Treatment options for stage I non-small-cell lung carcinoma patients not suitable for lobectomy. Expert Rev Anticancer Ther. 2009; 9:1443-1453.

29. Grills IS, Mangona VS, Welsh R, Chmielewski G, McInerney E, Martin S, Wloch J, Ye H, Kestin LL. Outcomes after stereotactic lung radiotherapy or wedge resection for stage I non-small-cell lung cancer. J Clin Oncol. 2010; 28:928-935.

30. Parashar B, Patel P, Monni S, Singh P, Sood N, Trichter S, Sabbas A, Wernicke AG, Nori D, Chao KS. Limited resection followed by intraoperative seed implantation is comparable to stereotactic body radiotherapy for solitary lung cancer. Cancer. 2010; 116:5047-5053.

31. Varlotto J, Fakiris A, Flickinger J, Medford-Davis L, Liss A, Shelkey J, Belani C, DeLuca J, Recht A, Maheshwari N, Barriger R, Yao N, DeCamp M. Matched-pair and propensity score comparisons of outcomes of patients with clinical stage I non-small cell lung cancer treated with resection or stereotactic radiosurgery. Cancer. 2013; 119:2683-2691.
32. Shirvani SM, Jiang J, Chang JY, Welsh JW, Gomez DR, Swisher S, Buchholz TA, Smith BD. Comparative effectiveness of 5 treatment strategies for early-stage nonsmall cell lung cancer in the elderly. Int J Radiat Oncol Biol Phys. 2012; 84:1060-1070.

33. Port JL, Parashar B, Osakwe N, Nasar A, Lee PC, Paul S, Stiles BM, Altorki NK. A propensity-matched analysis of wedge resection and stereotactic body radiotherapy for early stage lung cancer. Ann Thorac Surg. 2014; 98:1152-1159.

34. Matsuo Y, Chen F, Hamaji M, Kawaguchi A, Ueki N, Nagata Y, Sonobe M, Morita S, Date H, Hiraoka M. Comparison of long-term survival outcomes between stereotactic body radiotherapy and sublobar resection for stage I non-small-cell lung cancer in patients at high risk for lobectomy: A propensity score matching analysis. Eur J Cancer. 2014; 50:2932-2938.

35. Puri V, Crabtree TD, Bell JM, Broderick SR, Morgensztern D, Colditz GA, Kreisel D, Krupnick AS, Patterson GA, Meyers BF, Patel A, Robinson CG. Treatment Outcomes in Stage I Lung Cancer: A Comparison of Surgery and Stereotactic Body Radiation Therapy. J Thorac Oncol. 2015; 10:1776-1784.

36. Parashar B, Port J, Arora S, Christos P, Trichter S, Nori D, Wernicke AG. Analysis of stereotactic radiation vs. wedge resection vs. wedge resection plus Cesium-131 brachytherapy in early stage lung cancer. Brachytherapy. 2015; 14:648-654.

37. Paul S, Lee PC, Mao J, Isaacs AJ, Sedrakyan A. Long term survival with stereotactic ablative radiotherapy (SABR) versus thoracoscopic sublobar lung resection in elderly people: national population based study with propensity matched comparative analysis. BMJ. 2016; 354:i3570.

38. Safi S, Rauch G, op den Winkel J, Kunz J, Schneider T, Bischof M, Heussel CP, Huber PE, Herth FJ, Dienemann H, Hoffmann H. Sublobar resection, radiofrequency ablation or radiotherapy in stage I Non-small cell lung cancer. Respiration. 2015; 89:550-557.

39. Kent M, Landreneau R, Mandrekar S, Hillman S, Nichols F, Jones D, Starnes S, Tan A, Putnam J, Meyers B, Daly B, Fernando HC. Segmentectomy versus wedge resection for non-small cell lung cancer in high-risk operable patients. Ann Thorac Surg. 2013; 96:1747-1754.

40. Smith CB, Swanson SJ, Mhango G, Wisnivesky JP. Survival after segmentectomy and wedge resection in stage I nonsmall-cell lung cancer. J Thorac Oncol. 2013; 8:73-78.

41. Ezer N, Veluswamy RR, Mhango G, Rosenzweig KE, Powell CA, Wisnivesky JP. Outcomes after Stereotactic Body Radiotherapy versus Limited Resection in Older Patients with Early-Stage Lung Cancer. J Thorac Oncol. 2015; 10:1201-1206.

42. Zhao X, Qian L, Luo Q, Huang J. Segmentectomy as a safe and equally effective surgical option under complete videoassisted thoracic surgery for patients of stage I non-small cell lung cancer. J Cardiothorac Surg. 2013; 8:116. 
43. Nakamura H, Taniguchi Y, Miwa K, Adachi Y, Fujioka S, Haruki T, Takagi Y, Yurugi Y. Comparison of the surgical outcomes of thoracoscopic lobectomy, segmentectomy, and wedge resection for clinical stage I non-small cell lung cancer. Thorac Cardiovasc Surg. 2011; 59:137-141.

44. Okada M, Koike T, Higashiyama M, Yamato Y, KodamaK, Tsubota N. Radical sublobar resection for small-sized non-small cell lung cancer: a multicenter study. J Thorac Cardiovasc Surg. 2006; 132:769-775.

45. Gould MK, Kuschner WG, Rydzak CE, Maclean CC, Demas AN, Shigemitsu H, Chan JK, Owens DK. Test performance of positron emission tomography and computed tomography for mediastinal staging in patients with non-small-cell lung cancer: a meta-analysis. Ann Intern Med. 2003; 139:879-892.

46. De Ruysscher D, Wanders S, van Haren E, Hochstenbag M, Geeraedts W, Utama I, Simons J, Dohmen J, Rhami A, Buell U, Thimister P, Snoep G, Boersma L. Selective mediastinal node irradiation based on FDG-PET scan data in patients with non-small-cell lung cancer: a prospective clinical study. Int J Radiat Oncol Biol Phys. 2005; 62:988-994.

47. Hoopes DJ, Tann M, Fletcher JW, Forquer JA, Lin PF, Lo SS, Timmerman RD, McGarry RC. FDG-PET and stereotactic body radiotherapy (SBRT) for stage I nonsmall-cell lung cancer. Lung Cancer. 2007; 56:229-234.

48. Mancini BR, Park HS, Harder EM, Rutter CE, Corso CD, Decker RH, Husain ZA. Elderly patients undergoing SBRT for inoperable early-stage NSCLC achieve similar outcomes to younger patients. Lung Cancer. 2016; 97:22-27.

49. Parmar MK, Torri V, Stewart L. Extracting summary statistics to perform meta-analyses of the published literature for survival endpoints. Stat Med. 1998; 17:2815-2834.
50. Williamson PR, Smith CT, Hutton JL, Marson AG. Aggregate data meta-analysis with time-to-event outcomes. Stat Med. 2002; 21:3337-3351.

51. Tierney JF, Stewart LA, Ghersi D, Burdett S, Sydes MR. Practical methods for incorporating summary time-to-event data into meta-analysis. Trials. 2007; 8:16.

52. Matsuo Y, Nakamoto Y, Nagata Y, Shibuya K, Takayama K, Norihisa Y, Narabayashi M, Mizowaki T, Saga T, Higashi T, Togashi K, Hiraoka M. Characterization of FDG-PET images after stereotactic body radiation therapy for lung cancer. Radiother Oncol. 2010; 97:200-204.

53. Huang K, Dahele M, Senan S, Guckenberger M, Rodrigues GB, Ward A, Boldt RG, Palma DA. Radiographic changes after lung stereotactic ablative radiotherapy (SABR)-can we distinguish recurrence from fibrosis? A systematic review of the literature. Radiother Oncol. 2012; 102:335-342.

54. van Tulder M, Furlan A, Bombardier C, Bouter L, and the Editorial Board of the Cochrane Collaboration Back Review Group. Updated method guidelines for systematic reviews in the Cochrane Collaboration back review group. Spine. 2003; 28:1290-1299.

55. L'Abbe KA, Detsky AS, O'Rourke K. Meta-analysis in clinical research. Ann Intern Med. 1987; 107:224-33.

56. Dersimonian R, Laird N. Meta-analysis in clinical trials. Control Clin Trials. 1986; 7:177-88.

57. Begg CB, Mazumdar M. Operating characteristics of a rank correlation test for publication bias. Biometrics. 1994; 50:1088-101.

58. Egger M, Davey Smith G, Schneider M, Minder C. Bias in Meta-analysis detected by a simple, graphical test. BMJ. 1997; 315:629-34. 